\author{
Lei Meng \\ Song-Hua Fang \\ Mei Jin
}

\section{An unusual case of pancreatic and gastric neoplasms (2010: 12b)}

Received: 29 March 2010

Revised: 12 June 2010

Accepted: 21 June 2010

(C) European Society of Radiology 2011
M. Jin

Department of Pathology, Sir Run Run Shaw Hospital,

Sir Run Run Shaw Institute of Clinical Medicine of Zhejiang University,

Hangzhou, 310016 Zhejiang Province, China

\begin{abstract}
Gastrointestinal stromal tumors (GISTs) are the most common mesenchymal tumors of the digestive tract and are believed to originate from the neoplastic transformation of the interstitial cells of Cajal from their precursors. GIST can occur anywhere
\end{abstract}

but mainly affects the stomach $(60 \%)$, jejunum and ileum (30\%), duodenum (4-5\%), rectum (4\%), colon, appendix and esophagus. Primary GISTs of the pancreas are very rare, with only five previous cases reported. This is an unusual case of multiple GISTs in the pancreas and stomach.

Keywords Pancreatic neoplasms . Gastrointestinal stromal tumors . Stomach · Tomography, X-ray computed

\section{Case report}

A 42-year-old man incidentally found an upper abdominal mass 2 weeks previously. He denied any overt signs or symptoms of gastrointestinal bleeding. Full blood count and renal and liver functions were within normal limits.

On physical examination, an upper abdominal palpable mass was found. It was the size of a pigeon's egg, movable and soft, with no tenderness. He had no jaundice. He was not in acute distress, and his vital signs were normal.

He underwent ultrasound of his abdomen, which revealed a large cystic mass (Fig. 1a, arrow) in the pancreatic head with a thick capsule and a small septation. The mass measured $11.23 \times 7.25 \mathrm{~cm}$, and no significant blood flow signal was seen. A small amount of pancreatic tissue was demonstrated behind the cystic mass.

Unenhanced CT showed an ill-demarcated cystic mass (Fig. 1b, arrows) in the pancreatic head region with homogeneous density, while a small nodule (Fig. 1b, arrowhead) was seen in the gastric antrum with stippled calcification. On contrast-enhanced CT, a well-defined cystic mass (Fig. 1c, d, arrows) in the pancreatic head, measuring $11 \times 8 \mathrm{~cm}$, was found with irregular and nodular enhancement at the edge of the lesion, while a welldemarcated nodule $1.4 \mathrm{~cm}$ in diameter in the gastric antrum (Fig. 1d, arrowhead) was demonstrated with homogeneous and moderate enhancement.

Intraoperatively, a yellow mass measuring $1.5 \mathrm{~cm}$ in its maximum diameter extending outwards to the serosa was identified in the lesser curvature of the gastric antrum with intact mucosa. A cystic mass $10 \mathrm{~cm}$ in diameter, which had red-tan fluid, was found in the pancreatic head with a hypervascular capsule.

Gross pathological examination revealed two masses. One had gray-red soft tissue, measuring $10 \times 8 \times 3 \mathrm{~cm}$, and originated from the pancreas. A portion of the tissue was cystic with a thick capsule. Sectioning revealed dark red tissue with areas of cystic change. Under microscopic examination, the tumor showed spindle cells (Fig. 1e, black arrows) arranged in fascicles and whorls. The polynuclear tumor giant cells could be seen. The mitotic rate differed but was high in some areas with up to 5 mitotic figures per 50 high power fields (HPFs). There were extensive areas of necrosis (Fig. 1e, white arrows) and cystic degeneration. A small piece of pancreatic tissue was seen outside the capsule wall. Immunohistochemical study revealed positive staining for c-kit protein (Fig. 1f, arrow), but negative reactions to $\alpha$-smooth muscle actin and CD34. The other mass measuring $1.5 \mathrm{~cm}$ in its maximum diameter arose from the gastric antrum. There were osseous components and calcification was apparent 
Fig. 1 A 42-year-old man incidentally found an upper abdominal mass 2 weeks previously. a US reveals a large cystic mass in the pancreatic region (arrow). b Unenhanced CT shows ill-demarcated cystic mass between the liver and stomach with homogeneous density (arrow), while a small nodule is seen in the gastric antrum with stippled calcification (arrowhead). c On contrast-enhanced CT, a welldefined cystic mass (arrow) in the pancreatic head is found with irregular and nodular enhancement at the edge of the lesion. d Image reformatted in the coronal plane demonstrates a solid nodule (arrowhead) in the gastric antrum and a cystic mass in the pancreatic head region (arrow). e Under microscopic examination, the tumor shows spindle cells (black arrows) arranged in fascicles and whorls. There were extensive areas of necrosis and cystic degeneration (white arrows) $(\mathrm{HE} \times 100)$.

f Immunohistochemical study reveals positive staining for c-kit protein (arrow) $(\times 100)$. g Histologically, GIST arises from the muscularis propria of the stomach (arrow) $(\mathrm{HE} \times 10)$
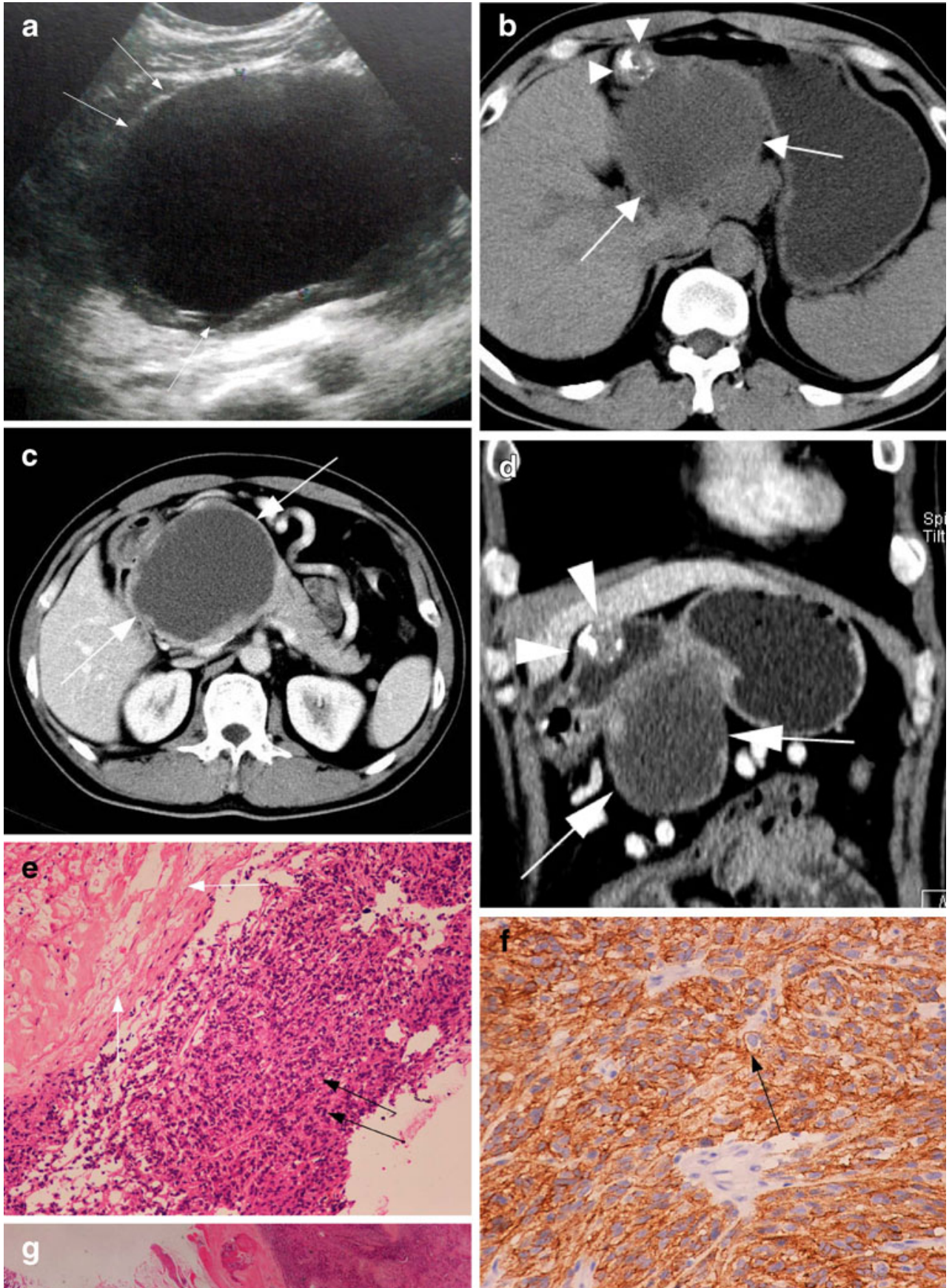
on gross examination. Histologically, the tumor showed spindle cells on the H\&E-stained tissue sections (Fig. 1g, arrow). The polynuclear tumor giant cells were also seen. The mitotic rate was high in some areas with up to 5 mitotic figures per 20 high power fields. Immunohistochemistry showed c-kit and SMA positivity. CD34, S-100, Des, and CK were negative. Based on the above findings, the tumors were finally diagnosed as malignant GISTs originating from the pancreas and stomach.

\section{Discussion}

Gastrointestinal stromal tumors (GISTs) are the most common mesenchymal tumors of the digestive tract and are believed to originate from the neoplastic transformation of the interstitial cells of Cajal from their precursors. Expression of CD117 (the c-kit receptor present in interstitial cells of Cajal) is seen in almost all GISTs and is therefore considered to be their defining feature. Approximately $70 \%$ of all GISTs are positive for the CD34 protein, which is a hematopoietic progenitor cell antigen, while $20-30 \%$ are positive for SMA, $10 \%$ are positive for S-100 protein, and fewer than $5 \%$ are positive for desmin, which is the intermediate filament protein of smooth, skeletal, and cardiac muscle cells [1]. GISTs can occur anywhere but mainly affect the stomach $(60 \%)$, jejunum and ileum $(30 \%)$, duodenum $(4-5 \%)$, rectum (4\%), colon, appendix, and esophagus [2]. The overall incidence has been estimated to be 10-20 per million. Primary GISTs are usually solitary, but can be multiple, as in Carney's triad or when associated with neurofibromatosis, but in our case, there was no evidence that there were GISTs co-existent with Carney's triad or neurofibromatosis. Primary GISTs of the pancreas are very rare, with only five previous cases reported [3, 4]. To our knowledge this is the first case of multiple GISTs in the pancreas and stomach described to date in the literature.

Clinically, the presentations of GIST are highly variable according to their size and the existence of mucosal ulceration. Small GISTs, especially those not accompanied by mucosal ulceration, are usually incidental findings upon operation, endoscopy, or imaging studies for other reasons, while large tumors are usually symptomatic, according to the previous report; these tumors most commonly present GI bleeding, abdominal pain, palpable mass, and intestinal obstruction. The tumor shows marked cystic transformation probably as a result of central necrosis and liquefaction, as we saw in our case.

Gastrointestinal stromal tumors have a wide clinicopathologic spectrum, ranging from small benign tumors to overt sarcomas. It is difficult to determine their malignant potential and prognosis. There has been extensive study of GIST to determine whether tumor size, mitotic activity, and genetic characteristics predict disease progression. GISTs more than $5 \mathrm{~cm}$ in size, independent of mitotic rate, are at moderate risk of metastases, and all tumors with more than 5 mitoses per 50 HPFs are at high risk of metastases.

Although very rare in the pancreas, gastrointestinal stromal tumors should be considered in the differential diagnosis of cystic pancreatic masses. Correct diagnosis of cystic GIST of the pancreas is very difficult before operation. It is often misdiagnosed as mucinous cystadenoma or pseudocyst. It may be helpful to note that the cystic GISTs have irregular and nodular enhancement at the edge of the lesion on contrast-enhanced CT. According to our case, if there is another GIST in the stomach or intestine, while the cystic-solid mass is found in the pancreas, the cystic pancreatic GIST should be considered first. In short, it is very difficult to differentiate cystic GIST from cystadenoma or cystadenocarcinoma in the pancreas based only on imaging findings, the final diagnosis is made by pathology.

\title{
References
}

1. Kwon SH, Cha HJ, Jung SW, Kim BC, Park JS, Jeong ID, Lee JH, Nah YW, Bang SJ, Shin JW, Park NH, Kim DH (2007) A gastrointestinal stromal tumor of the duodenum masquerading as a pancreatic head tumor. World $\mathbf{J}$ Gastroenterol 13(24):3396-3399
2. Miettinen M, Lasota J (2006) Gastrointestinal stromal tumors: pathology and prognosis at different sites. Sem Diagn Pathol 23(2):70-83

3. Showalter SL, Lloyd JM, Glassman DT, Berger AC (2008) Extra-gastrointestinal stromal tumor of the pancreas: case report and a review of the literature. Arch Surg 143(3):305-308
4. Yan BM, Pai RK, Van Dam J (2008) Diagnosis of pancreatic gastrointestinal stromal tumor by EUS guided FNA. JOP 9(2):192-196

\section{Precisely correct answer was received by closing date from:}

\author{
Manabu Minami, Ibaraki, Japan
}

